\title{
الكفاءة الاقتصادية لصناعة طحن القمح في مصر
}

\author{
ا I الدعادل حامد بهنساوى. \\ أستاذ ورئيس قسم الهندسة الزراعية ، جامعة بنها.

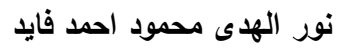 \\ طالبة دراسات عليا بقسم الاقتصاد الزراعى لئى
}

\author{
ا.د.محمد سعيد امين الثشتاوى \\ أستاذ الاقتصاد الزراعي، جامعة بنها

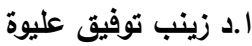 \\ أ.د كلية الاقتصاد والعلوم السياسية ، جامعة المستقبل
}

تعتبر صناعة طحن القمح في مصر من أهم الصناعات الزراعية باعتبار أن القمح ومنتجاتها الغذاء الرئيسى للغالبية العظمى من السكان ،

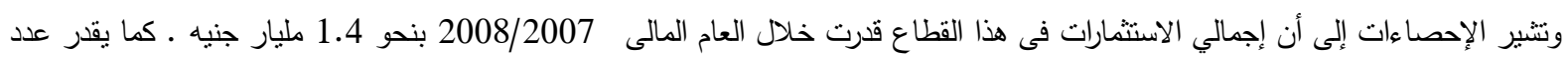

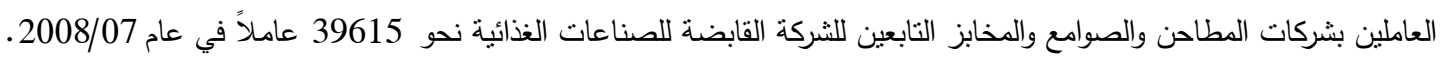

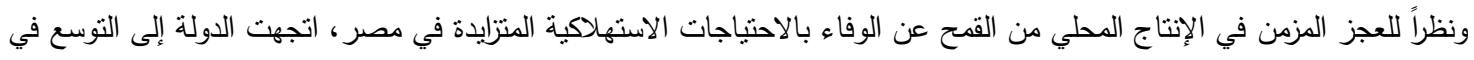

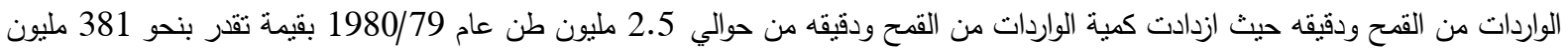

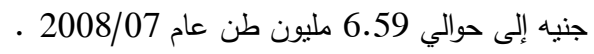

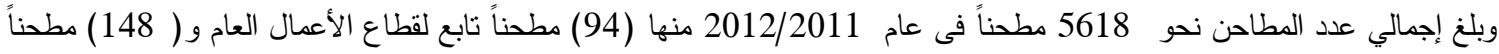

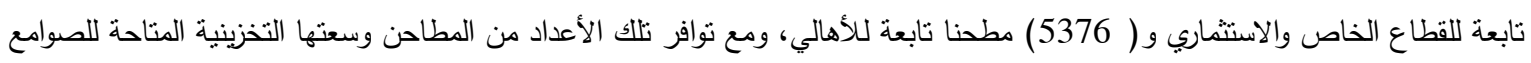

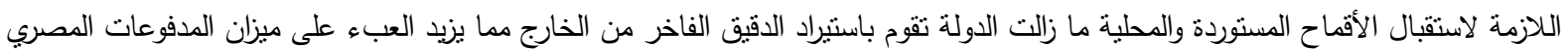
نتيجة لعدم التنتغيل الأمتل للطاقات الطاحنة وإهار رؤوس الأموال المستثمرة .

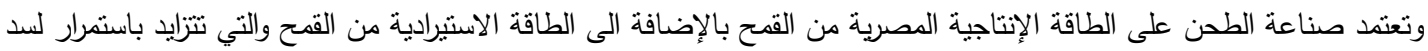

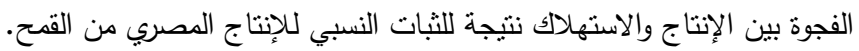

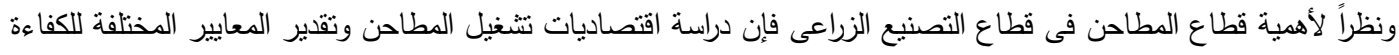

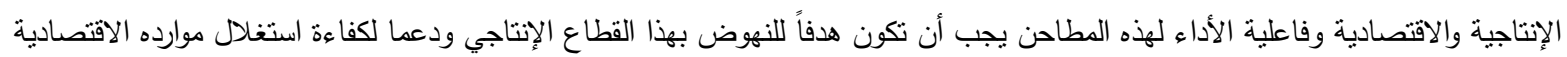
مما يؤهله للقيام بالدور المطلوب منه فى علاج الفجوة القحية التى تعانى منها جمهورية مصر العاد العربية.

مشكلة الداسة:

بالرغم من زيادة الإنتاج المحلى من القمح إلا أن الإنتاج من القمح مازال عاجز عن الوفاء بالاحتياجات الاستهلاكية الغذائية المتزايدة ،

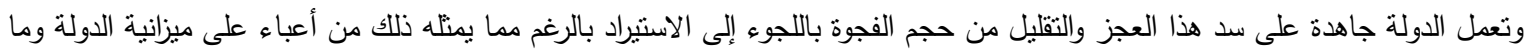

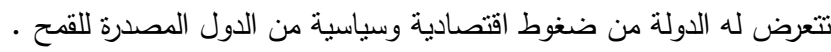

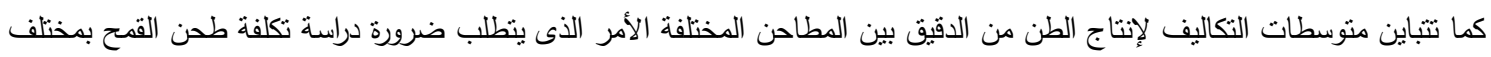

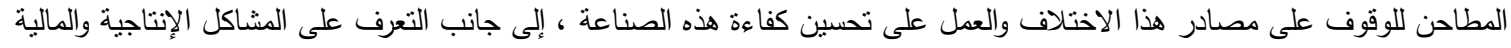

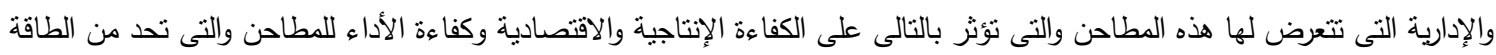

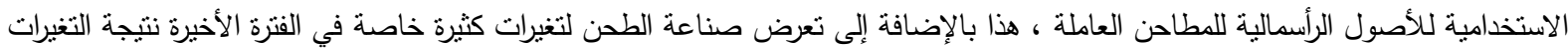

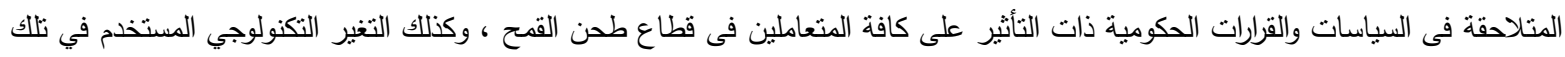

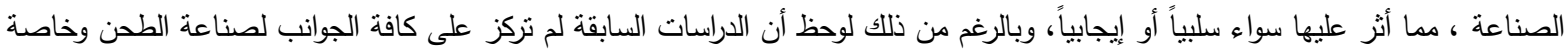

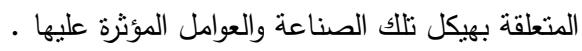

الهـف من الدراسة:

تهدف الدراسة إلى التعرف على اقتصاديات تصنيع القمح في شركة مطاحن شمال القاهرة ، والوقوف على الكفاءة الإنتاجية والاقتصادية

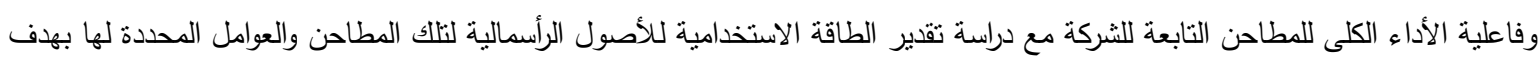

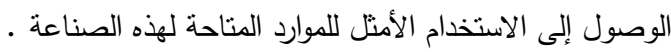


اعتمدت الدراسة لتحقيق أهدافها على استخدام أساليب التحليل الإحصائي الوصفى المتمنل في المتوسطات والنسب المئوية ومعدلات النمو التي استخدمت لتوصيف الظاهرة ، وأخيراً استخدام مقاييس الكفاءة سواء الإنتاجية أو التحويلية أو الاقتصادية، واستخدام مؤشرات تقييم فاعلية الأداء

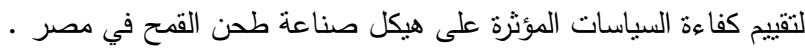

هذا وقد اعتمدت الدراسة على البيانات السنوية المنشورة وغير المنشورة من الجهات ذات الصلة بموضون الصنات الدراسة ، منل نشرات قطاع

الثئون الاقتصادية بوزارة الزراعة و الجهاز المركزي للتعبئة العامة والإحصاء، وهيئة السلع التموينية التابعة لوزارة التضامن الاجنماعي، والثركة

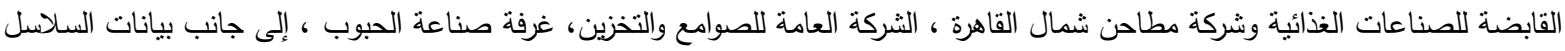
المقطعية الني تم الحصول عليها من قوائم التكاليف لميزانية شركة مطاحن شمال القاهرة ، وذلك لاستخدامها في التقدير الإحصائي لدوال التكاليف وتحديد السعة الإنتاجية المنلى لهذه المطاحن.

ونظراً لوجود صعوبات وعقبات فى الحصول على البيانات اللازمة من شركات الطحن التابعة للشركة القابضة للصناعات الغذائية فقد

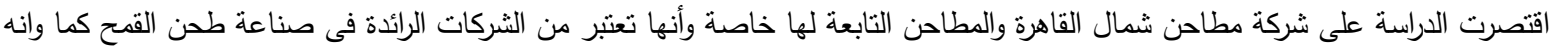
ينوفر بها أنواع مخنلفة من المطاحن .

مناقشة نتائج الدراسة نتتاول فيما يلى تقييم لنشاط طحن القمح بشركة مطاحن شمال القاهرة وذلك باستخدام أهم مؤشرات الكفاءة المستخدمة لتقييم صناعة طحن

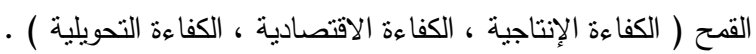
أولاً : الكفاءة الإنتاجية :

• الأهمية النسبية لنشاط الطحن بالنسبة لأنشطة شركة مطاحن شمال القاهرة

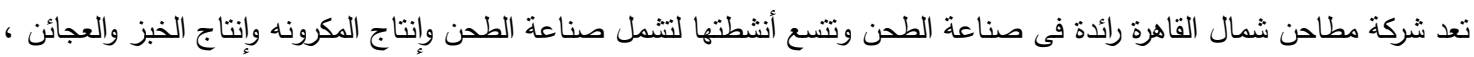

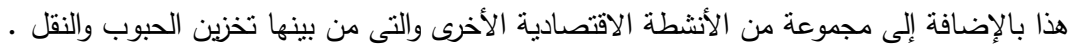

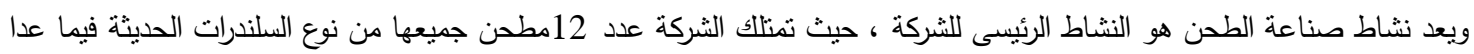
مطحن واحد يعمل بنظام القرص (الديسك ميل) لإنتاج الدقيق بجميع أنواعه .

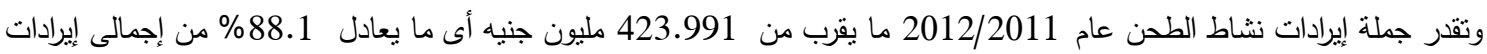
نثاط الثركة والبالغة نحو 481.1 مليون جنيه فى ذات العام ، أما النسبة المتنقية من الإيرادات فترجع لبقية الأثنطة . جدول رقم ( 1) : الأهية النسبية لإيرادات نشاط الطحن بالنسبة لباقى الأنشطة بشركة مطاحن شمال القاهرة خلال الفترة القيمة ألف جنيه (2012/2011

\begin{tabular}{|c|c|c|c|c|c|}
\hline \multirow{3}{*}{ متوسط الفترة } & \multicolumn{4}{|c|}{ السنوات } & \multirow{3}{*}{ البيان } \\
\hline & / 2011 & / 2010 & / 2009 & / 2008 & \\
\hline & 2012 & 2011 & 2010 & 2009 & \\
\hline 377895 & 423991 & 364650 & 342014 & 380923 & جملة إير ادات نشاط الطحن \\
\hline 89.0 & 88.13 & 88.47 & 90.62 & 88.86 & $\%$ \\
\hline 17978 & 26568 & 20122 & 9733 & 15489 & جملة إير ادات نشاط المكرونه \\
\hline 4.2 & 5.52 & 4.88 & 2.58 & 3.61 & $\%$ \\
\hline 751 & 1027 & 948 & 8.42 & 1019 & جملة إير ادات نشاط الخبز \\
\hline 0.2 & 0.21 & 0.23 & 0.22 & 0.24 & $\%$ \\
\hline 21905 & 21531 & 21111 & 20019 & 24960 & جملة إير ادات نشاط النقل الثقيل \\
\hline 5.2 & 4.48 & 5.12 & 5.30 & 5.82 & $\%$ \\
\hline 6033 & 7900 & 5252 & 4763 & 6218 & جملة إير ادات نشاط التخزين \\
\hline 1.4 & 1.64 & 1.27 & 1.26 & 1.45 & $\%$ \\
\hline 59 & 64 & 68 & 51 & 54 & جملة إير ادات مشغو لات للغير \\
\hline 0.01 & 0.01 & 0.02 & 0.01 & 0.01 & $\%$ \\
\hline 424829 & 481081 & 412151 & 377422 & 428663 & جملة إيراد النشاط \\
\hline
\end{tabular}


وبدراسة تطور الإيرادات المحققة وفقاً لأنشطة الثركة خلال الفترة 2012/2011-2009/2008 والذى توضحه بيانات الجدول رقم ( 1 (1)

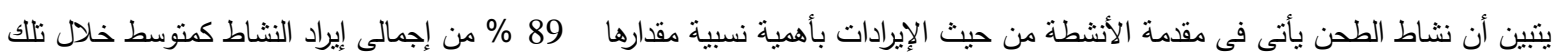

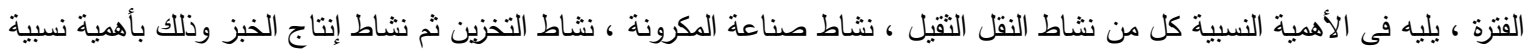

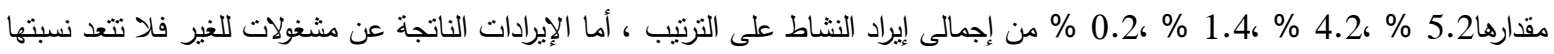

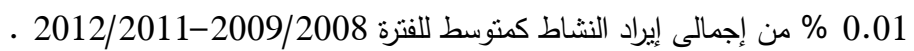
أما بالنسبة للأهمية النسبية لنكلفة نشاط الطحن بالنسبة لنكلفة بقية الأنثطة ، فنتير بيانات الجدول رقم (2) والذى يوضح الأهمية النسبية

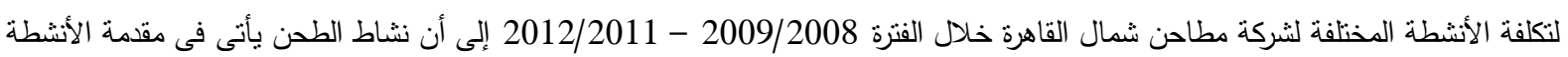
من حيث التكلفة بأهمية نسيية مقدارها 90.1 \% من إجمالى تكلفة النشاط كمتوسط لتلك الفترة ، يليه فى الأهمية النسبية كل من نثاط صناعة

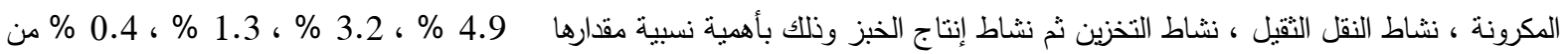
إجمالي تكلفة النشاط على الترتيب ، أما نكلفة مشغولات للغير فلا تتعد نسبتها 0.01 \% من إجمالي تكلفة النشاط كمتوسط للفترة

جدول رقم (2) : الأهمية النسبية لنكلفة نشاط الطحن بالنسبة لباقى الأنشطة بشركة مطاحن شمال القاهرة خلال الفترة (2009/2008(2012/2011

\begin{tabular}{|c|c|c|c|c|c|}
\hline \multirow{3}{*}{ منوسط الفترة } & \multicolumn{4}{|c|}{ السنوات } & \multirow{3}{*}{ البيان } \\
\hline & / 2011 & $/ 2010$ & / 2009 & $/ 2008$ & \\
\hline & 2012 & 2011 & 2010 & 2009 & \\
\hline 334998 & 366312 & 320834 & 307451 & 345394 & جملة تكلفة نشاط الطحن \\
\hline 90.1 & 88.31 & 89.46 & 91.65 & 91.34 & $\%$ \\
\hline 18379 & 27322 & 19861 & 11125 & 15209 & جملة تكلفة نشاط المكرونه \\
\hline 4.9 & 6.59 & 5.54 & 3.32 & 4.02 & $\%$ \\
\hline 1488 & 1901 & 1637 & 1169 & 1244 & جملة تكلفة نشاط الخبز \\
\hline 0.4 & 0.46 & 0.46 & 0.35 & 0.33 & $\%$ \\
\hline 11822 & 13524 & 11057 & 11178 & 11528 & جملة تكلفة نثاط النقل الثققيل \\
\hline 3.2 & 3.26 & 3.08 & 3.33 & 3.05 & $\%$ \\
\hline 5019 & 5687 & 5179 & 4502 & 4706 & جملة تكلفة نشاط التخزين \\
\hline 1.3 & 1.37 & 1.44 & 1.34 & 1.24 & $\%$ \\
\hline 50 & 52 & 58 & 44 & 46 & جملة تكلفة مشغو لات للغير \\
\hline 0.01 & 0.01 & 0.02 & 0.01 & 0.01 & $\%$ \\
\hline 371755 & 414798 & 358626 & 335469 & 378127 & جملة تكلفة النشاط \\
\hline
\end{tabular}

بيانات الشركة القابضة للصناعات الغذائية ، شركة مطاحن شمال القاهرة ، المركز المالى والحسابات الختامية ، سنوات مختلفة ، بيانات غير منشورة .

$$
\text { : مؤشرات الإنتاج " (n) }
$$

1. مدى تحقيق الثركة للأهداف الكمية لنشاط الطدن :

بدراسة بيانات الجدول رقم (3) والذى يوضح مدى تحقيق الثركة لأهدافها الرئيسية المتعلقة بالكميات المستهدف طحنها سواء كانت قحاً

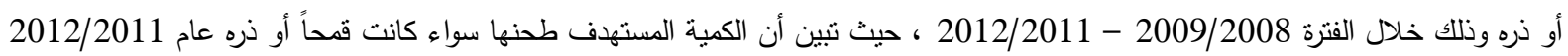

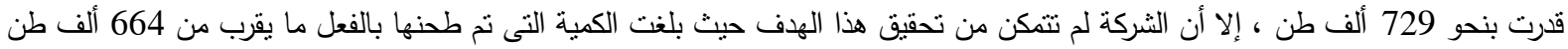
أى ما يعادل 91\% من الكمية المستهدف طنهها ، وقد برجع نلك إلى الربط المقرر على مطاحن الثركة من قبل وزارة التموين والتجارة الداخلية

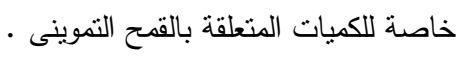


جدول رقم (3) : مدى تحقيق شركة مطاحن شمال القاهرة للأهداف الرئيسية المنعلقة ب نشاط الطحن خلال الفترة ( 2009/2008(2012/2011) القيمة:ألف طن كناف

\begin{tabular}{|c|c|c|c|c|c|}
\hline \multirow{3}{*}{ متوسط الفترة } & \multicolumn{4}{|c|}{ السنوات } & \multirow{3}{*}{ 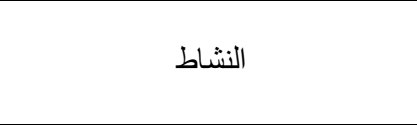 } \\
\hline & / 2011 & $/ 2010$ & / 2009 & $/ 2008$ & \\
\hline & 2012 & 2011 & 2010 & 2009 & \\
\hline 760.3 & 729 & 731 & 754 & 827 & الكمية المستهدف طحنها (قمح ، ذره ) \\
\hline 616.5 & 664 & 574 & 566 & 662 & الكمية الفعلية \\
\hline 81.1 & 91 & 78.5 & 75 & 80 & نسبة تحقيق المستهدف \% \\
\hline 121.8 & 120 & 116 & 116 & 135 & 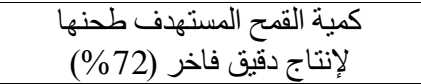 \\
\hline 60.5 & 69 & 73 & 53 & 48 & الكمية الفعلية \\
\hline 49.7 & 57.5 & 62.9 & 45.7 & 35.6 & نسبة تحقيق المستهدف \% \% \\
\hline
\end{tabular}

بيانات الثركة القابضة للصناعات الغذائية ، شركة مطاحن شمال القاهرة ، المركز المالى والحسابات الختامية ، سنوات مختلفة ، بيانات

وبدراسة تطور الكميات المستهدف طحنها من القمح والذزة والكميات التى تم طحنها بالفعل خلال الفتزة 2009/2008 - 2012/2011

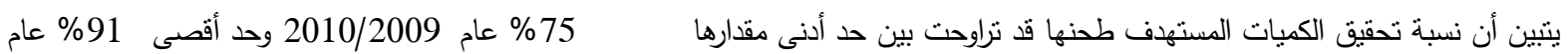

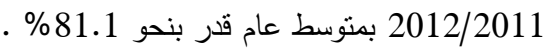

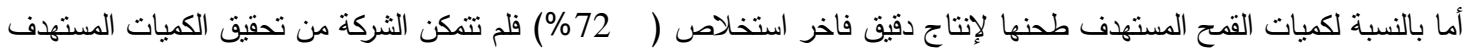
طحنها ، حيث لم تتككن الثركة سوى طحن ما يقرب من 57.5\% من الكمية المستهدف طحنها عام 2012/2011 والهقرة بنحو 120 ألف طن

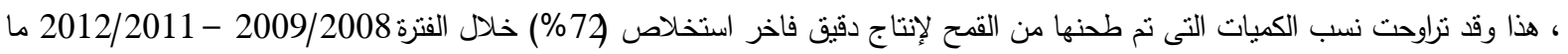
بين حد أدنى مقارها 35.6\% عام 2009/2008 وحد أقصى 62.9\% عام 2011/2010 ونلك من اجمالى الكميات المستهدفة وبمتوسط عام قدر بنحو 49.7\% ، وهذا ما يوضحه الجدول رقم (3).

\section{"تطور كميات القمح المطدون ومعدلات الإنتاج وفقاً لنوع الاقيق المنتج :}

بدراسة بيانات الجدول رقم (4) والذى يوضح تطور الكميات النى نم طحنها من القمح والذره وفقاً لنوع الدقيق المنتج وذلك خلال الفتزة 2009/2008 - 2012/2011 ، حيث يتضح أن إجمالى الكمية النى نم طحنها من القمح والذزة عام

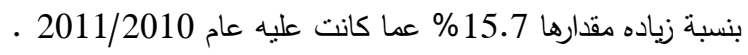

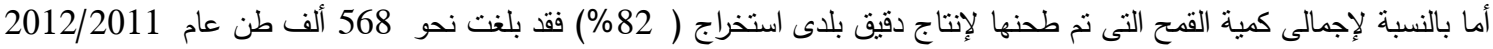

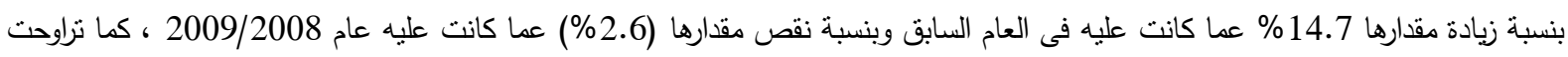

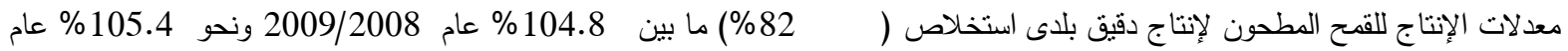
. 2012/2011

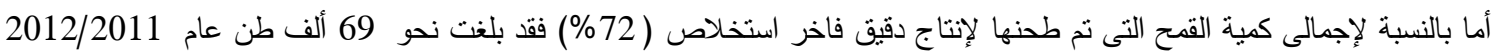

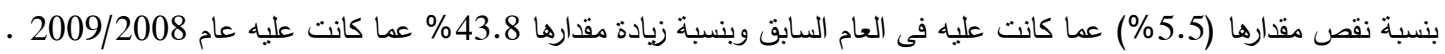

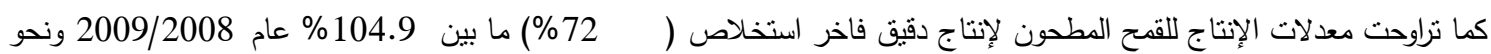

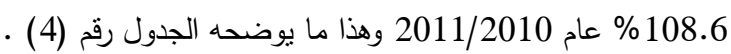

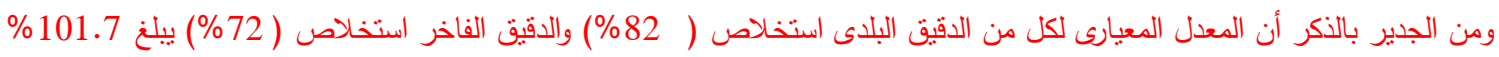

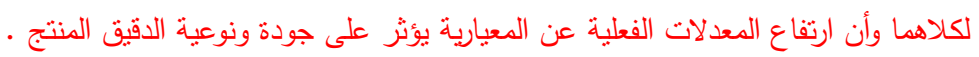

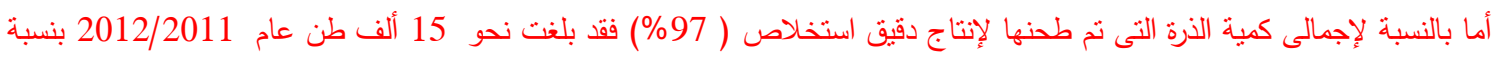
زيادة مقارها 150\% عما كانت عليه فى العام السابق وبنسبة نقص مقارها (51.6\%) عما كانت عليه عام 2009/2008 ، وهذا ما يوضحه 
جدول رقم (4) : نطور كميات القمح المطحون ومعدلات الإنتاج وفقاً لنوع الدقيق المنتج بشركة مطاحن شمال القاهرة خلال الفترة خلال الفترة (2012/2011-2009/2008)

\begin{tabular}{|c|c|c|c|c|}
\hline \multicolumn{4}{|c|}{ السنوات } & \multirow{3}{*}{ البيان } \\
\hline$/ 2011$ & $/ 2010$ & $/ 2009$ & $/ 2008$ & \\
\hline 2012 & 2011 & 2010 & 2009 & \\
\hline 652 & 574 & 566 & 662 & الكية المطحونة من القمح و الذرة \\
\hline 568 & 495 & 507 & 583 & كمية القمح المطحون لإنتاج دقيق بلدى استخلاص ( 82\%) \\
\hline 14.7 & $(2.4)$ & $(13.0)$ & - & 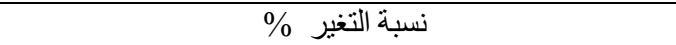 \\
\hline 105.4 & 105.3 & 105.2 & 104.8 & معدلات الإنتاج للقمح المطحون لإنتاج دقيق بلدى (82\%) \% \\
\hline 69 & 73 & 53 & 48 & كمية القمح المطحون لإنتاج دقيق فاخر استخلاص (72\%) \\
\hline$(5.5)$ & 37.7 & 10.4 & -- & 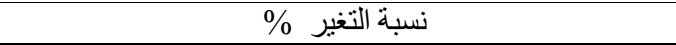 \\
\hline 105.3 & 108.6 & 105.6 & 104.9 & معدلات الإتتاج للقمح الدطحون لإنتاج دقيق فاخر (72\%) \% \\
\hline 15 & 6 & 6 & 31 & كمية الذرة المطحونة لإنتاج دقيق 97\% \\
\hline 150 & 00 & $(80.6)$ & -- & نسبة التغير \% م ك \\
\hline 101.7 & 101.7 & 101.7 & 101.7 & المعدل المعيارى \% \\
\hline
\end{tabular}

بيانات الثركة القابضة للصناعات الغذائية ، شركة مطاحن شمال القاهرة ، المركز المالى والحسابات الختامية ، سنوات مختلفة ، بيانات غير منشورة .

:الطاقات الإنتاجية المتاحة بالشركة ونسب استغلالها :

بدراسة بيانات الجدول رقم ( 5) الذى يوضح تطور الطاقة الإنتاجية المتاحة والطاقة الإنتاجية المستغلة بمطاحن الثركة وكنللك حجم

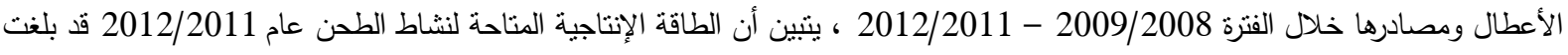
ما يقرب من 732 ألف طن بنسبة نقص مقارها ( 24.5\%) عما كانت عليه عام $2009 / 2008$ ـكما تنثير بيانات الجدول إلى أن الطاقة

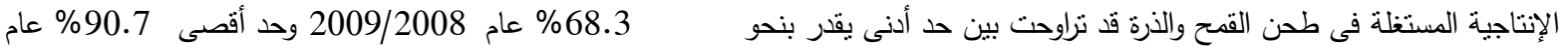
2012/2011 وذلك من اجمالى الطاقة الإنتاجية المتاحة .

جدول رقم (5) : تطور الطاقة الإنتاجية المستظة وحجم الأعطال ومصادر الأعطال لنثاط الطحن بشركة مطاحن شمال القاهرة خلال الفترة خلال الفترة (2009/2008-

\begin{tabular}{|c|c|c|c|c|}
\hline \multicolumn{4}{|c|}{ 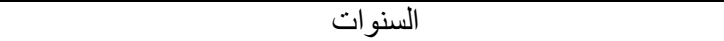 } & \multirow{3}{*}{ البيان } \\
\hline$/ 2011$ & $/ 2010$ & $/ 2009$ & $/ 2008$ & \\
\hline 2012 & 2011 & 2010 & 2009 & \\
\hline 732 & 731 & 741 & 969 & الطاقة الإنتاجية المتاحة لنشاط الطحن \\
\hline 664 & 574 & 566 & 662 & الكمية المطحونة (قصح ، ذره ) \\
\hline 90.7 & 78.5 & 76.4 & 68.3 & نسبة استغلال الطاقة الإنتاجية \% \\
\hline 126 & 202 & 195 & 210 & حجم الأعطال بالكمية لنشاط الطحن \\
\hline 17.2 & 27.5 & 26.3 & 21.7 & نسبة الأعطال للطاقة الإنتاجية المتاحة \% \\
\hline 75.7 & 73.1 & 81.6 & 76.4 & حجم الأعطال الراجعة للصبانة \\
\hline 60.1 & 36.2 & 41.8 & 36.4 & نسبة الأعطال الراجعة للصيانة \% \\
\hline 41.3 & 77.4 & 59.9 & 80.9 & حجم الأعطال الراجعة للتكس \\
\hline 32.8 & 38.3 & 30.7 & 38.5 & نسبة الأعطال الراجعة للتكدس \\
\hline 8.9 & 51.5 & 53.5 & 52.7 & حجم الأعطال الراجعة لأعطال أخرى \\
\hline 7.1 & 25.5 & 27.4 & 25.1 & نسبة الأعطال الراجعة لأعطال أخرى \% \\
\hline
\end{tabular}

بيانات الثركة القابضة للصناعات الغذائية ، شركة مطاحن شمال القاهرة ، المركز المالى والحسابات الختامية ، سنوات مختلفة ، بيانات غير منشورة .

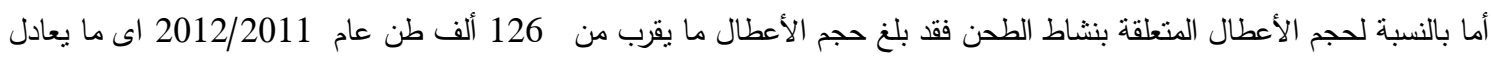

17.2 2002 من الطاقة الإنتاجية المناحة ، وتثثير البيانات إلى تناقص نسبة الأعطال مقارنة بحجم الطاقة الإنتاجية المتاحة ونلك خلال الفترة

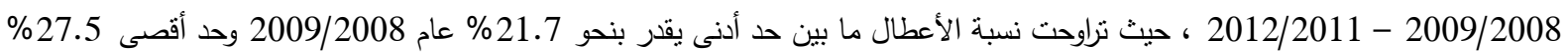

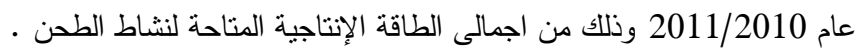


وتشبر البيانات إلى أن المصادر الرئيسية لتلك الأعطال تتحصر فى الصبانة ، التكس ، وأسباب أخرى متعددة ، أما بالنسبة للأعطال

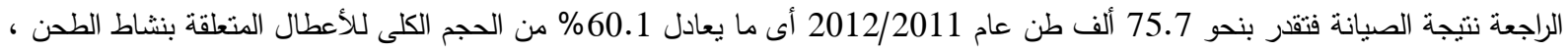

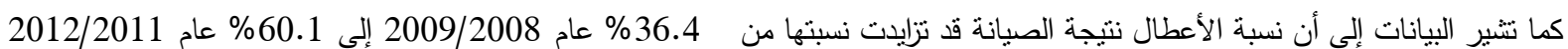
وذللك من اجمالى الطاقة المعطلة . أما بالنسبة للأعطال الراجعة للنكس فقد نتاقصت نسبتها من 38.5 \% عام 2009/2008 إلى نحو 32.8\% عام 2012/2011

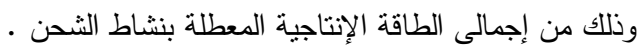
وتثتير البيانات إلى أن النسبة المتنقية من الأعطال ومقارها 7.1 \% عام 2012/2011 فترجع لأسباب أخرى متعددة ، ومن الملاحظ أن نسبة الأعطال الراجعة لأسباب أخرى قد تراجعت بشكل كبير فى العام الأخير حيث تراجعت نسبتها من 25.1\% عام الإن 2009/2008 إلى نحو

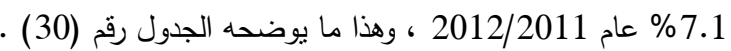

2. 2 - 2 الكفاءة الإنتاجية الكلية :

بدراسة بيانات الجدول رقم ( 6) والذى يوضح مؤشرات الكفاءة الإنتاجية لنشاط الطحن بشركة مطاحن شمال القاهرة خلال الفترة 2009/2008 - 2012/2011 يتبين أن الكفاءة الإنتاجية الكلية لنشاط الطحن بشركة مطاحن شمال القاهرة قد تراوحت بين

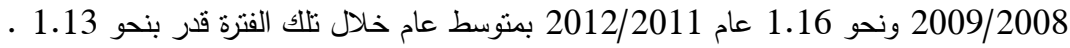

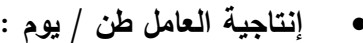

تثير بيانات الجدول السابق إلى أن انتاجية اليوم قد تزاوحت بين حد أدنى قدر بنحو 1856 طن عام 2010/2009 ونحو 2177

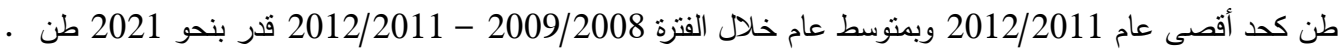

\section{إنتاجية الجنيه المستثمر :}

تنئير البيانات الواردة بالجدول السابق إلى أن إنتاجية الجنيه المستثر فى صناعة الطن بشركة مطاحن شمال القاهرة خلال الفترة 2009/2008 - 2012/2011 قد تراوح بين حد أدنى قلى بنحو 5.08 كجم/جنيه عام 2010/2009 ونحو 6.17 كجم/جنيه كحد أقصى عام 6 كام

2012/2011 وبمنوسط عام خلال الفترة 2009/2008 - 2012/2011 قدر بنحو 5.67 كجم/جنيه .

جدول رقم (6) : مؤشرات الكفاءة الإتتاجية لنشاط الطدن بشركة مطاحن شمال القاهرة خلال الفترة خلال الفترة (2009/2008- 2012/2011)

\begin{tabular}{|c|c|c|c|c|c|}
\hline \multirow{3}{*}{ منوسط } & \multicolumn{4}{|c|}{ 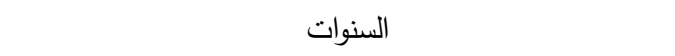 } & \multirow{3}{*}{ مؤشرات الكفاءة الإنتاجية } \\
\hline & / 2011 & / 2010 & / 2009 & / 2008 & \\
\hline & 2012 & 2011 & 2010 & 2009 & \\
\hline 1.13 & 1.16 & 1.14 & 1.11 & 1.10 & الكفاءة الإنتاجية الكلية \\
\hline 81.1 & 91 & 78.5 & 75 & 80 & نسبة تحقيق الخطة \% \\
\hline 2021 & 2177 & 1882 & 1856 & 2170 & إنتاجية اليوم طن/ يوم \\
\hline 0.55 & 0.61 & 0.52 & 0.50 & 0.57 & إنتاجية العامل طن/ يوم \\
\hline 5.67 & 6.17 & 5.43 & 5.08 & 5.99 & إنتاجية الجنيه المستثر كجم/ جنيه مستثر \\
\hline
\end{tabular}

بيانات الثركة القابضة للصناعات الغذائية ، شركة مطاحن شمال القاهرة ، المركز المالى والحسابات الختامية ، سنوات مختلفة ، بيانات غبر منشورة . n

ثانياً : الكفاءة الاقتصادية والتقييم المالى :

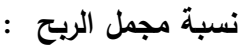

بدراسة بيانات الجدول رقم (7) والذى يوضح تطور مؤشرات الكفاءة الاقتصادية المستخدمة لتقييم صناعة طحن القمح لأنشطة شركة مطاحن شمال القاهرة ونلك خلال الفترة 2009/2008 - 2012/2011 ، حيث تبين أن نسبة مجمل الربح لأنثطة الثركة بلغت نحو 13.8 13. كمتوسط لتلك الفنرة ، وأن هذه النسبة قد تراوحت ما بين 11.89\% عام 2010/2009 ونحو 15.39\% كحد أقصى عام $2012 / 2011$ ، 
ونتير البيانات إلى أن هذه النسبة متزايدة من عام لأخر فيما عدا عام 2010/2009 الأمر الذى يثير إلى أن نسبة نمو مجمل الربح يفوق نسبة

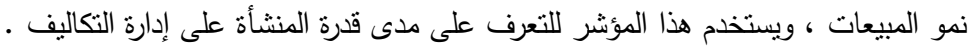

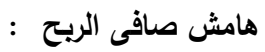

هذا المعيار يعبر عن نسبة صافي الربح إلى صافي المبيعات ويستخدم هذا المعيار فى التعرف على مدى قدرة الثركة على تحقيق

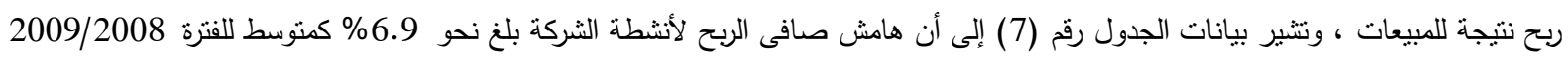

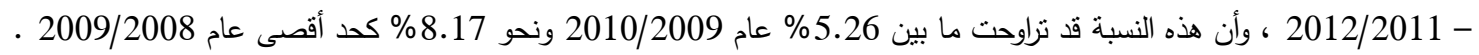

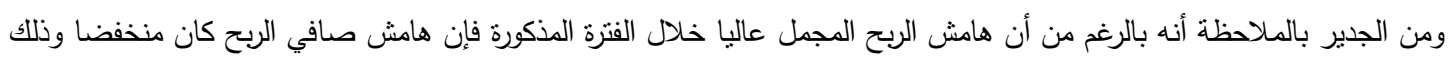

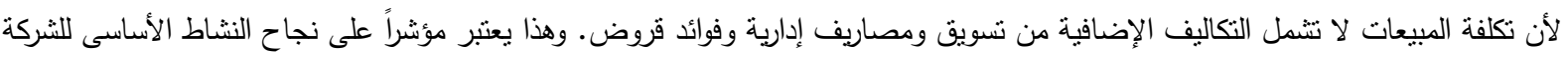

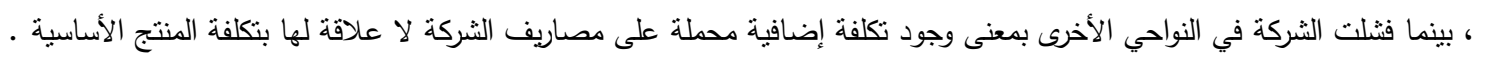

معل العائد على حقوق المساهمين (حقوق الملكية) : وهو نسبة صافي الربح إلى منوسط حقوق المساهمين هذا المعيار يعبر عن نسبة العائد على الاستثمار المتمثل فى حقوق المساهمين

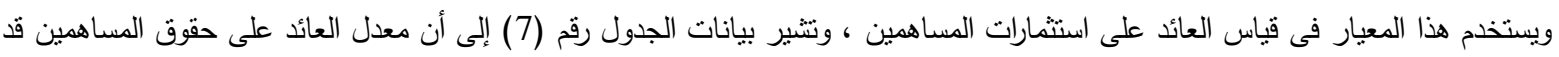

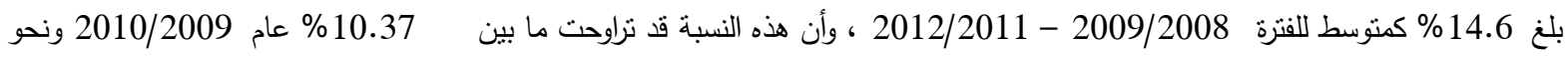

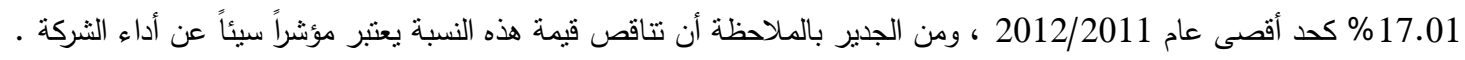
جدول رقم ( 7 ) : نطور مؤشرات الكفاءة المستخدة لنقييم صناعة طحن القمح بشركة مطاحن شمال القاهرة خلال الفترة خلال الفترة (2012/2011-2009/2008)

\begin{tabular}{|c|c|c|c|c|c|}
\hline \multirow{3}{*}{ متوسط الفترة } & \multicolumn{4}{|c|}{ 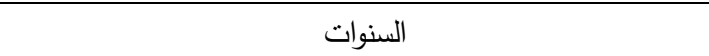 } & \multirow{3}{*}{ المؤشرات } \\
\hline & / 2011 & / 2010 & / 2009 & / 2008 & \\
\hline & 2012 & 2011 & 2010 & 2009 & \\
\hline 13.8 & 15.39 & 15.03 & 11.86 & 12.92 & نسبة مجمل الربح \\
\hline 6.9 & 7.31 & 6.81 & 5.26 & 8.17 & هامش صافى الربح \\
\hline 14.6 & 17.01 & 14.17 & 10.37 & 16.71 & معدل العائد على حقوق المساهمين \\
\hline 10.5 & 12.11 & 10.43 & 7.35 & 12.15 & معدل العائد على الأصول \\
\hline 24.5 & 28.52 & 22.56 & 17.21 & 29.68 & معدل العائد على الاستثمار \\
\hline 27.6 & 30.90 & 26.54 & 20.52 & 32.33 & صافى الربح على راس المال \\
\hline 238.2 & 237 & 250.7 & 225.1 & 239.8 & معدل التداول % \\
\hline 29 & 20.3 & 28.8 & 28.6 & 38.4 & نسبة السيوله السريعة \% \\
\hline
\end{tabular}

بيانات الشركة القابضة للصناعات الغذائية ، شركة مطاحن شمال القاهرة ، المركز المالى والحسابات الختامية ، سنوات مختلفة ، بيانات غير منشورة - من

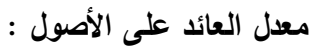
وهو نسبة صافي الربح إلى إجمالي الأصول (أي مجموع الأصول المنداولة والثابتة) ، ويستخدم هذا المعيار فى قياس مدى مساهمة

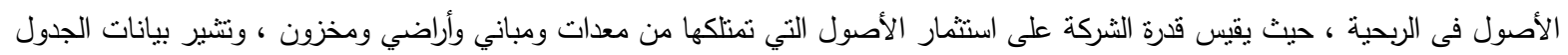

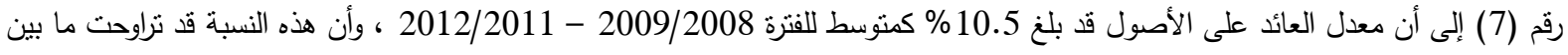

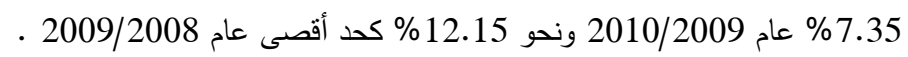

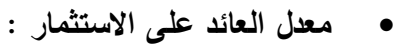
وهو نسبة صافي الربح إلى إجمالي الاستثمار ، ويستخدم هذا المعيار فى قياس العائد على اجمالى الاستثمار ، وتثير بيانات الجدول رقم

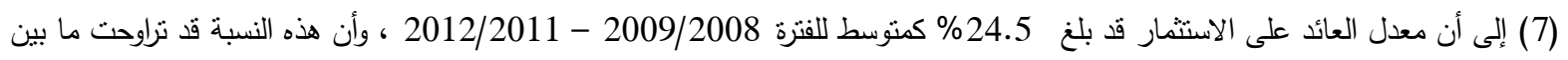

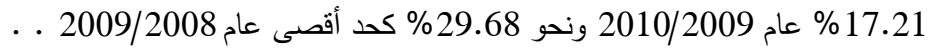




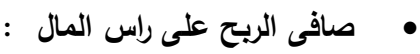

هذا المعيار يعبر عن نسبة صافي الربح إلى راس المال العامل ويستخدم هذا المعيار فى قياس قدرة رأس المال العامل الربحية ، ونتئير

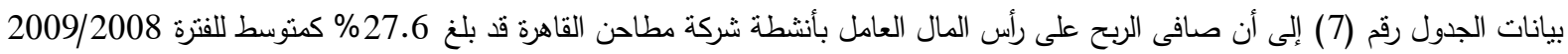

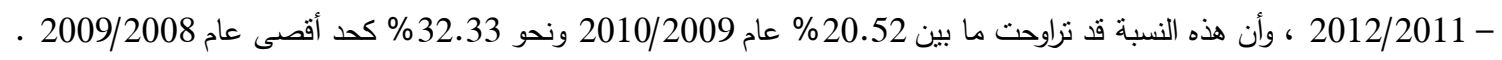

هذا المعيار يعبر عن نسبة الأصول المتداولة إلى مجموع الالتزامات المتداولة ، ويستخدم هذا المعيار فى التعرف على هدى قدرة الثركة

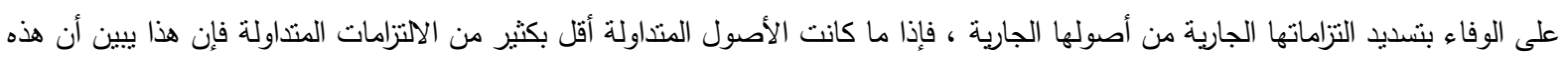
الثركة ستواجه مشاكل في سداد التزاماتها.

\section{: :}

هذا المعيار يعبر عن نسبة الأصول المتداولة السريعة إلى مجموع الالتزامات المتناولة ، وتعتبر هذه النسبة مشابهة لمعدل التداول غير انها

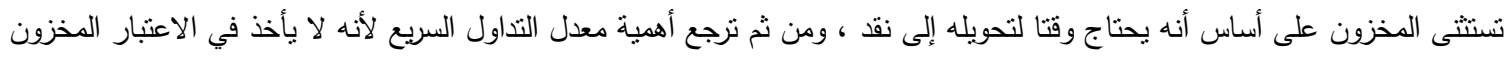

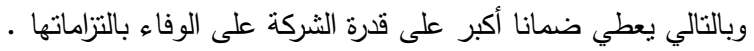
ثالثا : مراقبة الجودة في المطحن

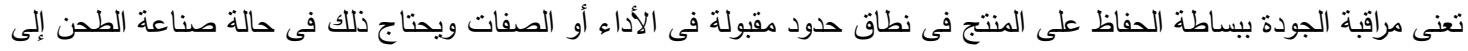
اهتمام جميع العاملين بدءاً من المختصين باستقبال القمح وتخزينه وحتى القائمين بالتعبئة النهائية للادقيق وتوزيعه .

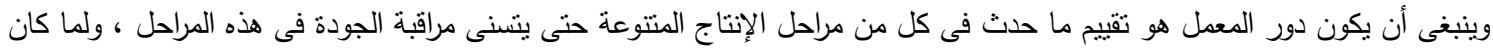

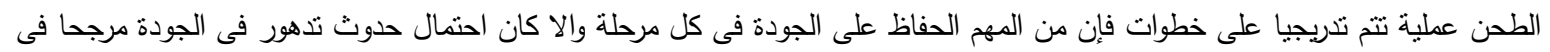
جميع المراحل التالية .

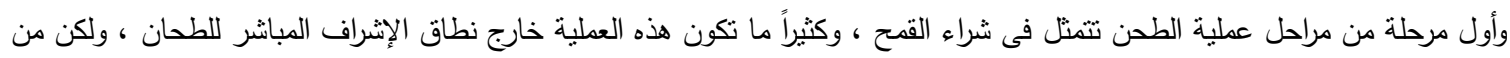

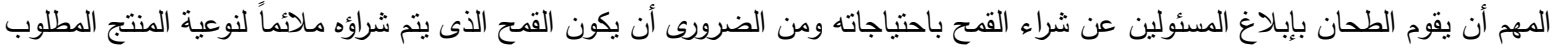

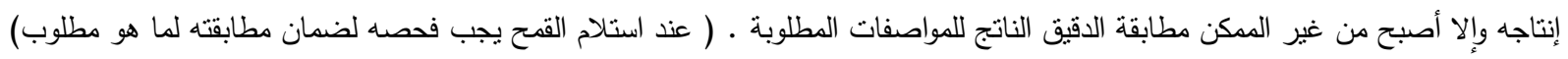
وتثمل بعض الاختبارات التى تجرى على القمح ما يلى : visual inspection moisture protein impurities wheat hardness kernel size milling test baking test

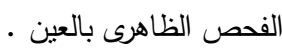

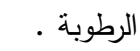
· البروتين نسبة الشوائب انبرون

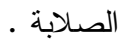

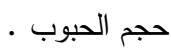

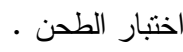

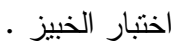

ويلزم اخذ مجموعة كاملة من العينات من القمح الوارد لاكتثاف اى تفاوت داخل الثحنة ، وتتسم هذه الخطوة بأهية خاصة بالنسبة اللبروتين والرطوبة وصلابة الحبوب وينبغى قدر الامكان ضغط التفاوت إلى أدنى حد عن طريق الخلط أو التقليب الكامل للحبوب قبل التبل التكييف

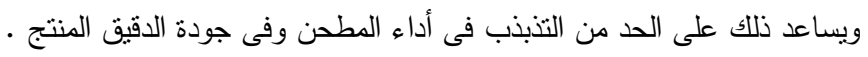

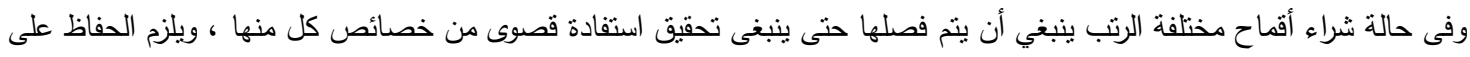

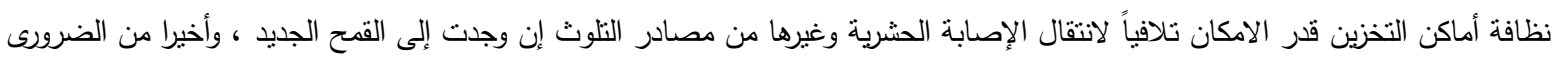

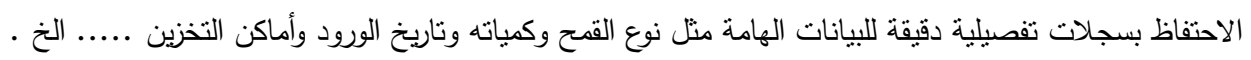

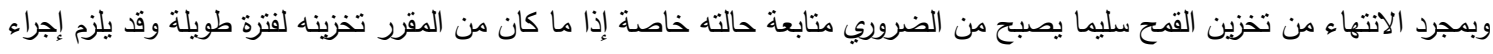

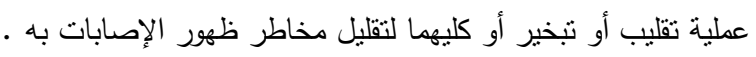

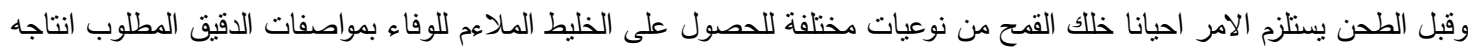

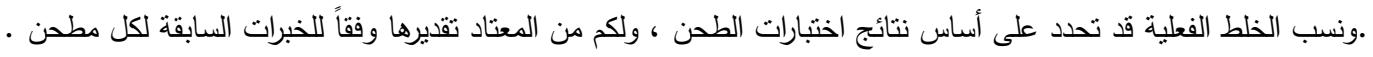


وينم بعد ذللك تتظيف القمح الخليط بقسم التنظيف ثم تكييفه .

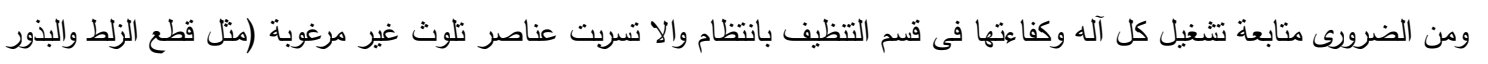

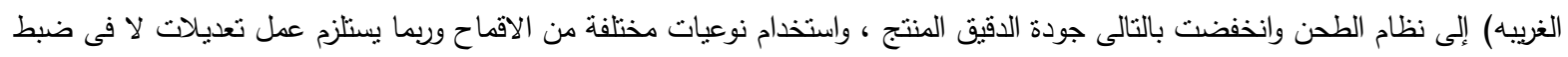

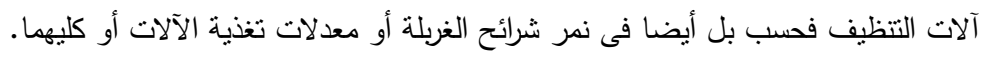

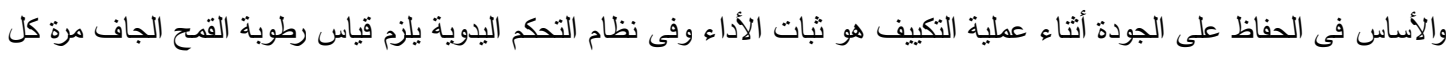

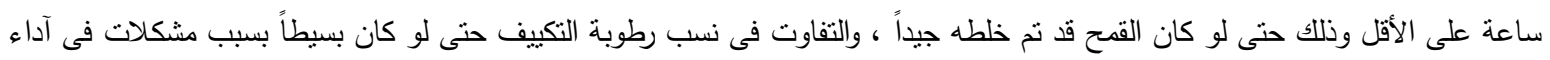

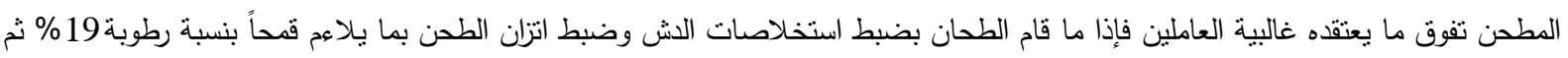

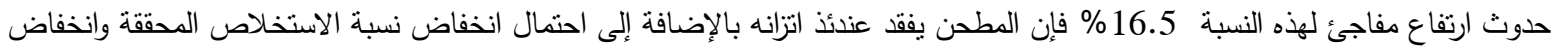

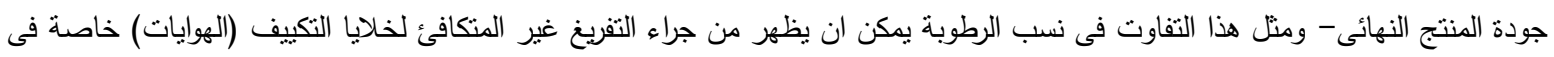

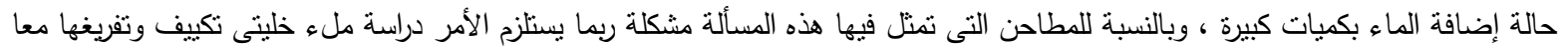

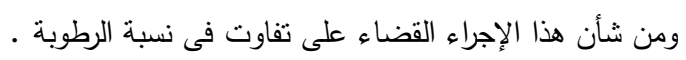

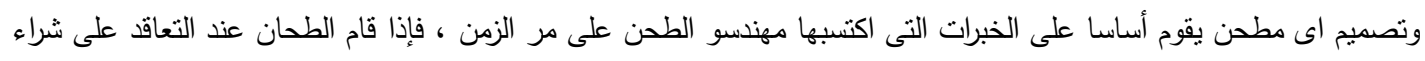

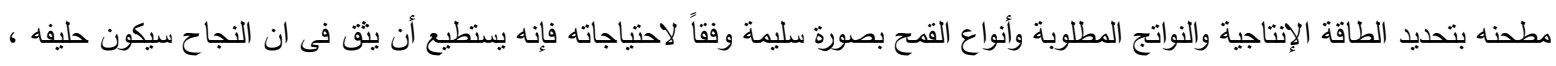

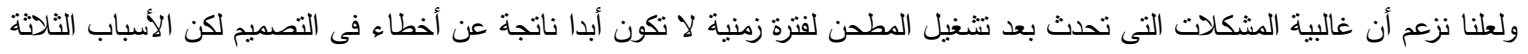
الأكثر احتمالاً لظهور هذه المشكلات هى :-

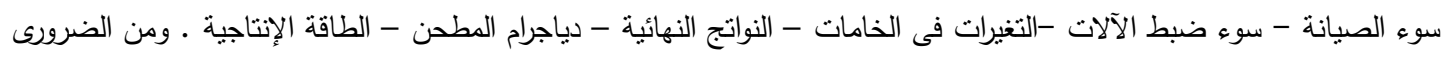

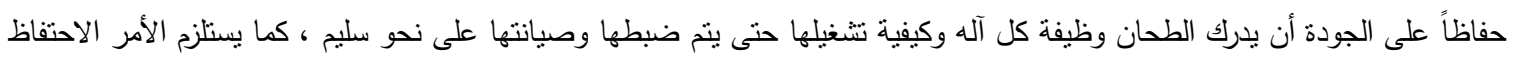

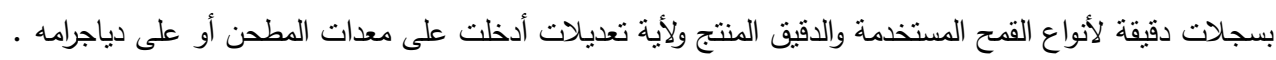

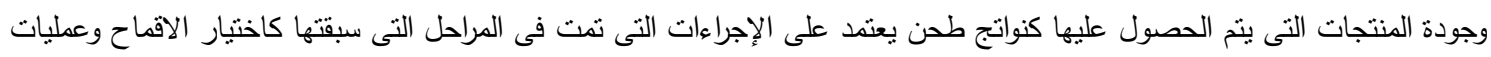
النظافة والخط .

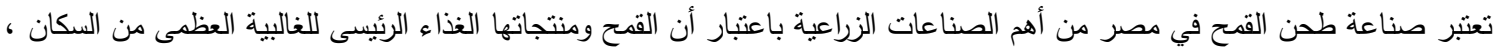

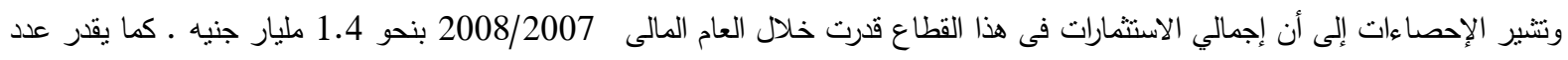

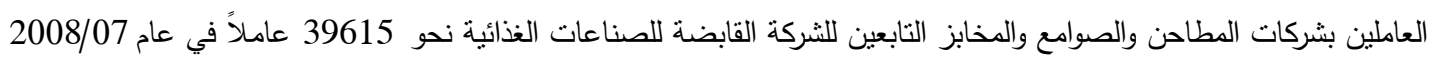

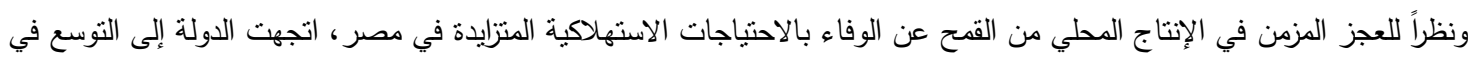

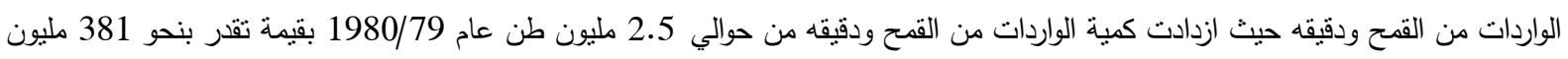

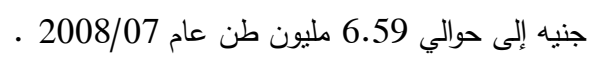

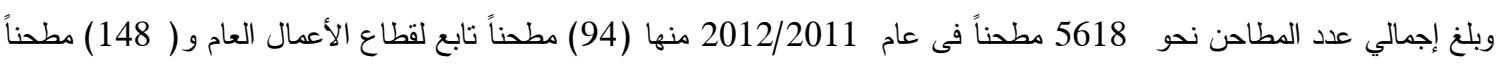

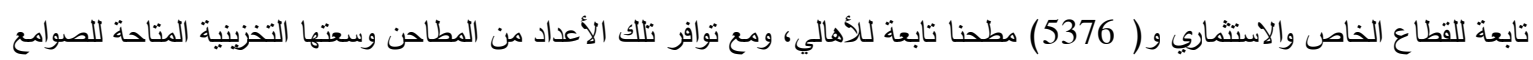

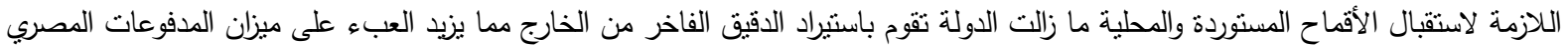

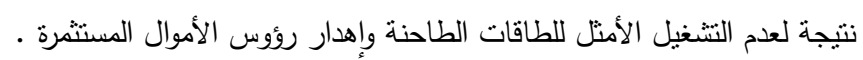

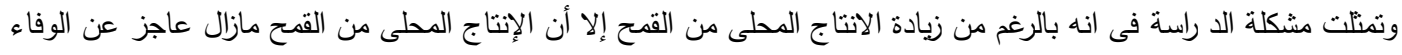

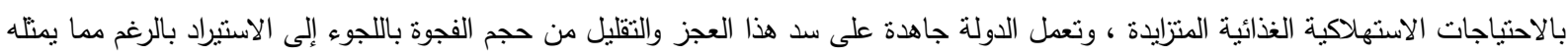

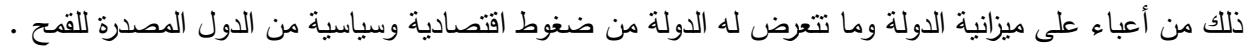

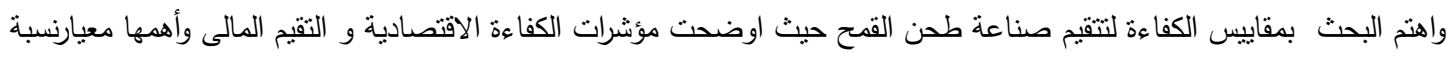

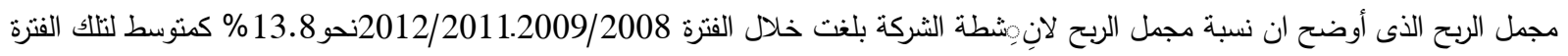

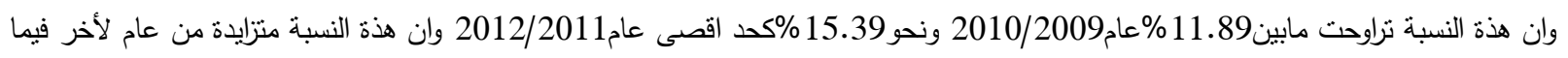

عدا عام 2010/2009\% ويشبر ذلك الى ان نسبة مجمل الربح يفوق نسبة نمو المبيعات. وقد نم استخدام بعض من معايرالكفاءة ومنها

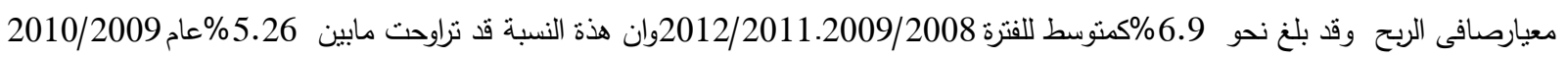

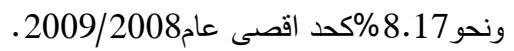


- زيادة المخزون الاستراتيجي للقمح من ثلاثة اثهر الى ستة اثشهر لتلافى تقلبات الاسواق العالمية.

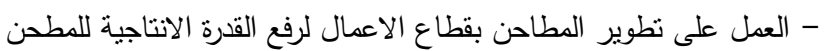
- العمل على اتمام انثاء مشروع الخمسين صومعة وعدم الاعتماد على الثنون الفرعية. - أنشاء قسم خاص لتعليم الطحن بالتعليم الزراعى لتقديم كوادر فنية لهذه الصناعة ألناء - العمل على نقل المطاحن فى منطقة بعيدة عن المناطق السكنية حفاظا على البيئة.

المراجع

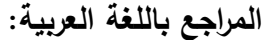

احمد ابو اليزبد عبد الرسول ( دكتور) و آخرون ؛ بعض الملامح الاقتصادية لاعم و إنتاج و استهلاك الخبز البلدى بمحافظة

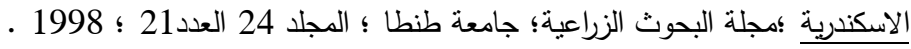

احمد عبد الغفار (دكتور ) ، السياسات الإنتاجية والسعربة والتسويقية للقمح فى جمهورية مصر العربية ، الجمعية المصرية للاقتصاد الزراعى

، المؤتمر الثالث للاقتصاديين الزراعيين ، 2-3 فبراير ، القاهرة ، 1994 .

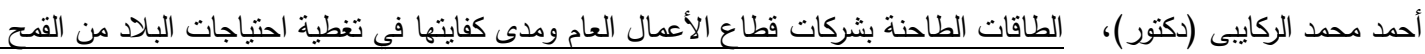

واستخداماته المختلفة، المؤتمر الأول لإستراتيجية إنتاج القمح المصري في ظل المتغيرات العالمية، 2008

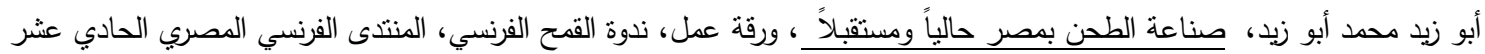

للحبوب، 2008.

جمال محمد عبد العزيز قيود، اقتصاديات صناعة طحن القمح في ج.م.ع مع التركيز على شركة مطاحن وسط وغرب الدلتا، رسالة دكتوراه،

قسم الاقتصاد الزراعي، كلية الزراعة بكفر الثيخ، جامعة طنطا، 2005.

حسن مبروك حسن زيدان ، اقتصاديات صناعة طحن القمح في مصر ، رسالة ماجستير ، قسم الاقتصاد الزراعي ، كلية الزراعة ، جامعة

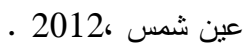

اتحاد الصناعات المصربة ، غرفة صناعة الحبوب ومنتجاتها، التقرير السنوي لشعبتي الطحن والخبز ، تقارير سنوات متعددة .

اتحاد الصناعات المصرية ، غرفة صناعة الحبوب ومنتجاتها ، مجلة الحبوب ، اعداد متفرقة .

الثركة القابضة للصناعات الغذائية ، شركة مطاحن شمال القاهرة ، المركز المالى والحسابات الختامية ، سنوات مختلفة ، بيانات غير

منشورة.

الشركة القابضة للصناعات الغذائية، الإدارة العامة للتخطيط، دراسة تحليلية عن نطور القدرات الطاحنة والتوجهات المستقبلية لإنتاج الدقيق

التمويني 82\% ومدى وفائها بالطلب عليها بشركات مطاحن قطاع الأعمال العام، دراسة غير منشورة، 2005.

وزارة الزراعة واستصلاح الأراضي , قطاع الثئون الاقتصادية , الإدارة المركزية للاقتصاد الزراعي، نشرة الاقتصاد الزراعي , أعداد مختلفة.

ثانياً: : المراجع الأجنبية

1.Heady, E.O. and J. L. Dillon : Agricultural Production Functions. IOW states university press, Ames, IWOA, 1961, p 73.

2. Left wich , R . H . , " The Price System and Resource Allocation " Revised Edition Holt , Rinehart and Winstion .N.Y . 1961, pp 136-137.

3.F.A.O., "Trade ear Book" different volumes.

4.http://faostat.fao.org/site/567/DesktopDefault.aspx?PageID=5674. (8/27/2009)

5 . http://www.egynews.net/wps/portal/news?params=78506\# (27/10/2009). 


\title{
The Economic Efficiency of Wheat Milling Industry in Egypt
}

\author{
Prof. Dr. Mohamed S. A. El- Sheshtawy *, Prof. Dr. Adel H. Bahnasay **, Prof. Dr.Zenab T. Elewa ***, \\ Nour El Hoda M. A. Fayed* \\ * Department of Agri. Economics, Faculty of Agriculture- Banha University \\ ** Department of Agri. Engineering, Faculty of Agriculture- Banha University \\ **** Faculty of Economic, Future University
}

\begin{abstract}
Summery
The wheat milling industry in Egypt of the most important agro-industrial manufacturing, and they play an important role in achieving many of the national goals in all stages of economic and social development, with total investments implemented until 07/2008 worth some 1.4 billion pounds.

The total number of about 5618 mill grinders in 2011/2012, including 94 mill belonging to the public business sector and 148 mill continued continued private sector investment and 5376 mill belonging to the people.The milling industry depend on the production capacity in addition to the Egyptian energy Alostradah of wheat, which is constantly growing to fill the gap between production and consumption as a result of the repayment of the Egyptian Egypt's wheat production.
\end{abstract}

Research Problem: The research problem in contrast to the average cost to produce a ton of flour among the various mills. Which requires the need to examine the cost of milling wheat for various mills to stand on this difference and work to improve the efficiency of the industry and to identify the problems of productivity and financial and administrative faced by the mills, Which affect the productivity and economic efficiency and efficient performance of the mills, which limit the power of usability of capital assets.

Objective of the research: The research aims to identify the economics of manufacturing wheat at North Cairo Mills, and stand on the production efficiency and economic effectiveness of the overall performance of the mills of the company and study estimating energy usability of the capital of these mills and their determinants in order to optimize the use of available resources for the industry.

Research method and data sources : The researcher on secondary data from published and unpublished from the relevant authorities goods subject of the study, which was obtained from the publications sector Affairs Economic Ministries of Agriculture and the Central Agency for Public Mobilization and Statistics and SCA at the Ministry of Social Solidarity, the Holding Company for Food Industries, Chamber of grain industry, which is beside CT series data obtained from the lists of costs to the budget of North Cairo Flour Mills Company, to be used in the statistical estimation of cost functions and determine the optimal production capacity of these mills. 